\title{
Belgeo
}

Revue belge de géographie

\section{Les institutions internationales, révélatrices des dynamiques régionales. Pour une lecture géographique de l'espace aérien européen et de son intégration}

International Institutions, revealing Regional Dynamics. For a Geographic

Approach of European Airspace and its Integration

\section{Antoine Beyer}

\section{(2) OpenEdition}

\section{Journals}

\section{Édition électronique}

URL : http://journals.openedition.org/belgeo/43572

DOI : $10.4000 /$ belgeo.43572

ISSN : 2294-9135

\section{Éditeur :}

National Committee of Geography of Belgium, Société Royale Belge de Géographie

\section{Référence électronique}

Antoine Beyer, «Les institutions internationales, révélatrices des dynamiques régionales. Pour une lecture géographique de l'espace aérien européen et de son intégration », Belgeo [En ligne], 4 | 2020, mis en ligne le 09 novembre 2020, consulté le 11 novembre 2020. URL : http://

journals.openedition.org/belgeo/43572 ; DOI : https://doi.org/10.4000/belgeo.43572

Ce document a été généré automatiquement le 11 novembre 2020.

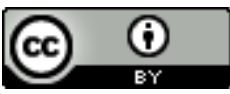

Belgeo est mis à disposition selon les termes de la licence Creative Commons Attribution 4.0 International. 


\section{Les institutions internationales,} révélatrices des dynamiques régionales. Pour une lecture géographique de l'espace aérien européen et de son intégration

International Institutions, revealing Regional Dynamics. For a Geographic

Approach of European Airspace and its Integration

Antoine Beyer

\section{Introduction. L'espace aérien envisagé dans la perspective de l'intégration régionale}

1 En complément d'une géographie du transport aérien qui s'intéresse d'abord aux dimensions technico-économiques (Vowles, 2006; Bowen, 2010) ou qui envisage l'espace dans le déploiement des stratégies des compagnies aériennes (Strale, 2006; Théry, 2017) et les effets de recomposition des marchés (Dobruszkes, 2014), nous abordons pour notre part l'espace aérien sous l'angle juridico-politique du contrôle (Sanguin, 1977; Marc, 2014) (Frétigny, 2013a, 2013b) que le phénomène de régionalisation soumet à un processus singulier de territorialisation.

2 La gestion de l'espace aérien européen relève simultanément de différents organismes qui cherchent, chacun à sa manière, à répondre aux besoins de sécurité et d'efficacité technique et économique des trafics, au-delà des seules prérogatives nationales. Comment alors expliquer un tel foisonnement? Leurs entreprises sont-elles concurrentes, complémentaires ou bien encore hiérarchisées ? La multiplicité d'acteurs est-elle transitoire ou pérenne? Que nous dit cette coexistence, au-delà de la seule gestion de l'espace aérien, sur la construction même d'un ensemble politique régional? 
Partant de ces interrogations, notre propos cherche à montrer comment les organismes internationaux en charge de l'encadrement de l'espace aérien ont été successivement mis en place et appelés à se réagencer au gré des évolutions politiques et institutionnelles.

L'approche retenue ici renvoie fondamentalement à la notion de souveraineté dans la lignée des travaux de John Agnew $(2005,2009)$ ou de Keith G. Debbage (2014) qui évoque la "géopolitique du transport aérien » et l'envisage dans la succession des systèmes de régulation (regulatory regimes) dont l'État territorial est la pièce maîtresse. Les juristes du droit communautaire des transports ont abordé de manière nuancée la façon dont les relations institutionnelles s'agencent. Ils sont d'un grand secours pour aborder les contours de la territorialité de l'espace aérien dans une dynamique d'européanisation (Grard, 2003 ; Correia, 2014).

Dans une perspective géographique de territorialisation régionale, les institutions qui encadrent les systèmes de transport sont paradoxalement peu mobilisées par les géographes alors même qu'ils expriment de nouveaux équilibres territoriaux (Beyer, 2008, 2014, 2019). Leur interprétation dans une démarche de spatialisation constitue pourtant un apport fort utile. Elle suppose en retour que l'analyse géographique s'ouvre à une approche multidisciplinaire, aussi bien vers le droit que vers l'histoire. L'article s'appuie sur un corpus de la littérature académique en droit, histoire et en géographie ainsi que sur la littérature grise concernant la gestion de l'espace aérien. Il en propose une réinterprétation à l'aune de l'intégration régionale (Partie 1). Derrière une apparente continuité de dénomination, les institutions aériennes fonctionnent comme des indicateurs des évolutions géopolitiques et des phases successives d'intégration. Partant des institutions existantes, nous avons donc cherché à en comprendre la diversité par le contexte de leurs genèses (Partie 2) afin de mettre ensuite en lumière les réorganisations systémiques qu'impliquent les dynamiques récentes d'intégration qu'une synthèse graphique et cartographique essaie de visualiser (Partie 3). La synthèse proposée sous forme de cartes et de graphiques est un apport inédit qui permet de mieux saisir l'interaction entre les diverses institutions en charge de la gestion du ciel européen. Du fait de leurs spécificités et pour ne pas alourdir un texte déjà dense, les singularités potentielles des régions périphériques de l'UE n'ont pas été abordées dans ce texte.

\section{Les lectures critiques de l'espace européen des transports : la legal geography au prisme de l'histoire des institutions internationales}

5 Le croisement du droit et de la géographie dans une perspective pluridisciplinaire est relativement récent (Forest, 2009, 2015). D'abord développé avec la «legal geography» en tant que champ de recherche en Amérique du Nord, il s'est diffusé en France à la faveur d'une territorialisation accrue de l'action publique. Les textes législatifs et les règlements interviennent en effet de plus en plus directement dans la fabrication des espaces dans les domaines de l'urbanisme ou de l'environnement. Les perspectives de la globalisation des échanges et le renouvellement de la question de la maîtrise des frontières par les États dans un contexte de rupture technologique renforcent cette convergence (cf. Journal of Borderland Studies). La géographie s'orientant plus explicitement vers une lecture des rapports de pouvoir dans l'espace ne pouvait qu'être 
interpellée par l'étude des rapports spatiaux-normatifs que porte le Droit (Blomley, 1994).

Notre hypothèse est que l'approche juridique des institutions en charge de la gestion des espaces aériens nous informe sur la structuration spatiale des grands ensembles régionaux. Elle rejoint de ce fait l'interrogation des géographes et peut conduire à de fructueux échanges interdisciplinaires dans la relation dialectique entre contrôle de l'espace et normes juridiques. L'approche intéresse aussi la communauté des géographes à la recherche d'une clé de compréhension du fait régional (Richard, Mareï, 2018), alors que le postulat qui reconnaît l'État territorial comme seul sujet du droit international est précisément questionné dans le droit international contemporain par l'émergence des ensembles régionaux.

7 Depuis plusieurs années, des historiens néerlandais (Erik van der Fleuten, 2005 ; Johan Schot, 2010, 2011) et suédois (Arne Kaijser, 2005) regroupés au sein du réseau Transnational Infrastructures and the Rise of Contemporary Europe ainsi que du groupe d'historiens allemands de l'université de Siegen autour de Christian Heinrich-Franke $(2007 \mathrm{a} / \mathrm{b}, 2008,2012)$ ont orienté leurs recherches sur la constitution d'une Europe des transports entre 1850 et 1950. L'originalité de leur approche repose justement sur l'importance trop méconnue des institutions internationales. Aussi invoquent-ils une « dimension cachée de l'intégration européenne " (Thomas et al., 2005), dans le sens où ces dernières sont sous-évaluées au profit des seules structures communautaires. Or, dès avant le traité de Rome de 1957 ou l'Acte unique de 1986 qui marque le début d'une politique communautaire des transports (Decoster, Versini, 2009), l'émergence d'institutions a contribué sur le long terme à jeter les bases de la construction régionale contemporaine (Schot, 2011; Stevens, 2004). Entrepris pour le rail, la route, la voie d'eau, cette lecture n'a été réalisée que partiellement pour l'aérien (Heinrich-Franke, 2008).

\section{La succession des institutions gestionnaires de l'espace aérien}

\section{Le contexte d'émergence du droit aérien international}

8 Le droit international assure aux État la pleine souveraineté sur l'espace aérien audessus de leurs territoires terrestres ou maritimes jusqu'à 12 miles nautiques $(22 \mathrm{~km})$ des côtes ; ils sont ainsi libres d'en contrôler l'accès. Au sein de cet espace s'exercent les règles de l'État survolé, alors qu'en haute-mer s'appliquent les règles de l'OACI (Organisation de l'aviation civile internationale, institution spécialisée des Nations Unies établie pour gérer la Convention de Chicago de 1944) ${ }^{1}$. La souveraineté s'exprime aussi par un droit de visite et de contrôle sur les appareils étrangers (avions et licences des pilotes) qui sont tenus de respecter le choix des aéroports pouvant les accueillir ou permettre leur départ. Les aéronefs sont placés sous l'autorité du pays dont il battent pavillon national, selon le principe d'immatriculation A ce titre, ils sont considérés comme extension du territoire national avec l'exigence du respect des règles et du contrôle technique de cet État.

9 Néanmoins, pour répondre aux évolutions techniques, singulièrement dans le domaine des transports aériens dont le rayon d'action n'a cessé de croître, les États ont émis des principes communs de circulation et créé des organisations internationales pour en 
réguler les trafics. Les bases de coordination jetées par les Accords de Paris (1910) ont abouti à la Convention de Paris (1919). Dans le sillage de la Conférence de la Paix dominée par les Alliés, est fixé le principe d'une souveraineté aérienne calqué sur le droit maritime. La CINA (Commission internationale de Navigation Aérienne) voit alors le jour, placée sous la responsabilité nominale de la SDN (Pelsser, 2019). Parmi les 33 signataires (sur les 50 États souverains de l'époque), l'absence des États-Unis, de la Chine et de l'URSS après sa création conférait à ce texte une coloration très européenne étendue aux espaces coloniaux. La résolution de problèmes opérationnels a donné lieu à des groupes de travail restreints ${ }^{2}$ qui soulignent d'emblée le lien entre projets régionaux et gestion interétatique de l'espace aérien international.

Au sortir de la Seconde Guerre mondiale, l'ordre aérien international est unifié autour de l'OACI. Il reprend le principe de souveraineté complète et exclusive des États sur leurs espaces aériens ${ }^{3}$ et confère un caractère strictement interétatique au droit international de l'aviation civile (Grard, 2003). Les questions d'interopérabilité technique et de transmission d'information (conditions atmosphériques, emplacement, équipement, état et condition d'ouverture des aérodromes) sont envisagées dans un cadre multilatéral (Guinchard, 1961). L'essentiel de ce travail se retrouve dans la définition de normes et de pratiques recommandées par l'OACI ${ }^{4}$. Pour leur part, les éléments commerciaux relèvent de seuls accords bilatéraux entre les États d'origine et de destination du vol. Ils sont établis sur la base de l'échange des quatre premières libertés aériennes dans un cadre du "système bermudien ", en référence au premier accord signé en 1946 aux Bermudes entre les États-Unis et le Royaume-Uni et qui a servi de modèle pour les suivants.

\section{L'accumulation des structures européennes de coopération}

11 Ce système initial à deux échelles (global pour les éléments techniques de navigation aérienne et bi-étatique pour les questions commerciales du transport) va aussi susciter la création d'espaces régionaux multilatéraux, démarche que l'OACI soutient comme le précise l'article 55a de sa charte ${ }^{5}$, favorisant l'émergence d'une échelle intermédiaire. L'OACI elle-même s'appuie sur des structures régionales au nombre de six, dont l'EANP (European Navigation Planning Group) mis en place en 1972 et qui regroupe tous les pays d'Europe (et après la chute du Mur, les États issus de l'ancienne URSS), ainsi que certains pays au sud du bassin méditerranéen (pays du Maghreb, Turquie et Israël). Leurs travaux sont réalisés en coordination avec les États concernés (56 pour l'EANP) depuis le bureau régional de Paris. Les PIRGs (Planning and Implementation Regional Groups) qui en sont issus visent à transcrire, à harmoniser et à actualiser les règles générales du contrôle aérien à l'échelle régionale sur une base intergouvernementale. Avant 1972 déjà, d'autres structures placées sous l'égide de l'OACI, traitaient de questions semblables. Il est alors intéressant de voir quelles ont été les étapes et les logiques institutionnelles à l'œuvre dans la constitution de cette échelle intermédiaire.

12 La création successive de structures marque de fait les différents moments de la construction politique européenne. Elle met en jeu des éléments techniques, des considérations juridiques et politiques qu'il est nécessaire de mettre en perspective pour comprendre la genèse $d u$ système aérien actuel. La différence entre ces institutions réside cependant moins dans leur objet, qui est très général, celui de la sécurité et de l'efficacité des conditions de vol, que dans la variation de leur étendue 
géographique et de l'intensité même de la coopération. Quatre institutions à vocation européenne se sont succédées et coexistent aujourd'hui encore. Elles relèvent de deux grandes deux principes: le régionalisme coopératif ou intergouvernemental avec la CEAC (Conférence Européenne de l'Aviation Civile) et les JAA (Joint Aviation Authorities) et le régionalisme intégratif avec Eurocontrol et l'AESA (Agence européenne de sécurité aérienne). Cette dualité conduit à établir un clivage important qu'il faut chercher à repérer pour voir comment s'établit un nouvel équilibre juridique et partant, une nouvelle forme pour l'espace aérien européen lui-même (fig. 1).

Figure 1. Schéma interprétatif de la généalogie du système aérien régional européen.

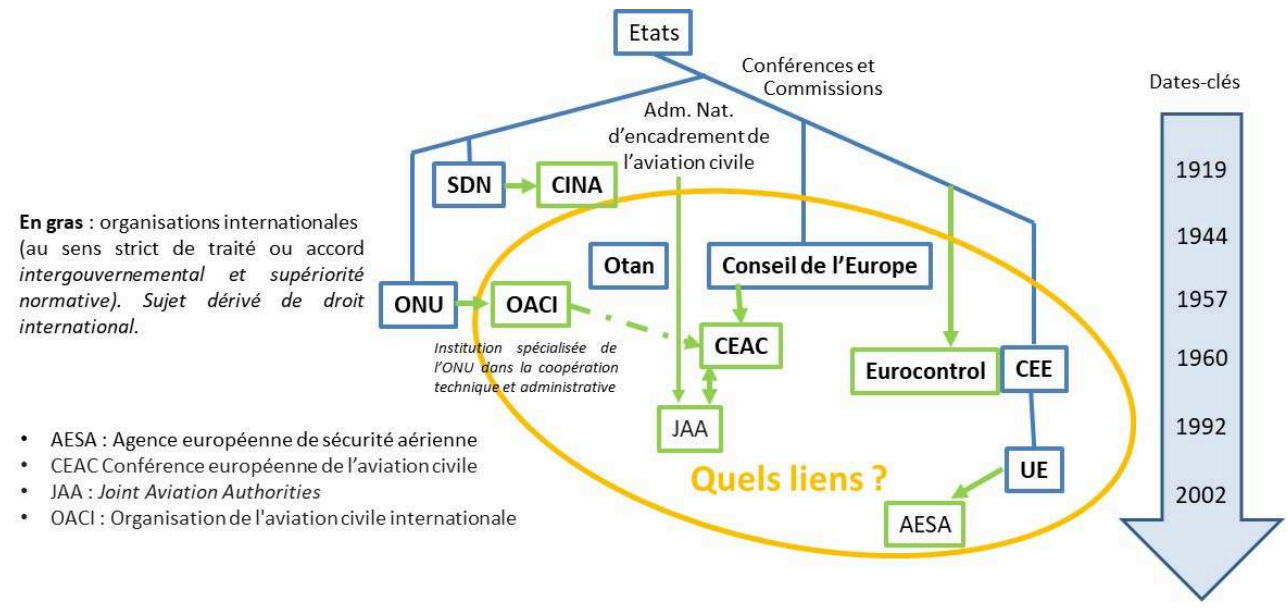

Conception : A. Beyer

\section{La CEAC (1955), dans le sillage de l'OACI}

La première organisation à se mettre en place est la CEAC à laquelle adhèrent 19 pays fondateurs en 1955. L'initiative en revient à l'Assemblée du Conseil de l'Europe ${ }^{6}$ qui propose au Comité des ministres de pousser le plus loin possible la coordination des Etats dans le domaine aérien. En l'absence de vues convergentes, l'initiative est placée sous l'égide de l'OACI qui en accepte le parrainage (Henrich-Franke, 2007b), d'autant qu'elle-même militait pour la création de structures régionales pour les plans de navigation aérienne (définition de routes, équipements, procédures) (Guinchard, 1961). La CEAC est ainsi conçue comme le cadre d'échanges d'informations entre organismes nationaux sur les questions aériennes et est appelée à devenir le lieu d'harmonisation et d'adaptation des normes universelles préconisées par l'OACI. Ses contours ne correspondent pourtant pas aux découpages régionaux beaucoup plus amples établis ultérieurement par l'OACI. La CEAC se modèle sur une réalité géopolitique qui oppose alors en Europe les États de l'ouest, membres du Conseil de l'Europe aux États de l'est qui n'y adhérent qu'après la chute du rideau de fer (fig. 2). La CEAC fixe de manière autonome son programme de travail et anime des conférences et des sessions qui lui sont propres. Ceci ne l'empêche pas de travailler en étroite collaboration avec l'OACI et son secrétariat (Vaugeois, 2014) où elle promeut les positions européennes. L'adoption des textes et recommandations y sont prises à l'unanimité, ce qui garantit le principe de souveraineté de ses membres. 
Figure 2. Les élargissements successifs de la CEAC et d'Eurocontrol (avec la date d'adhésion).
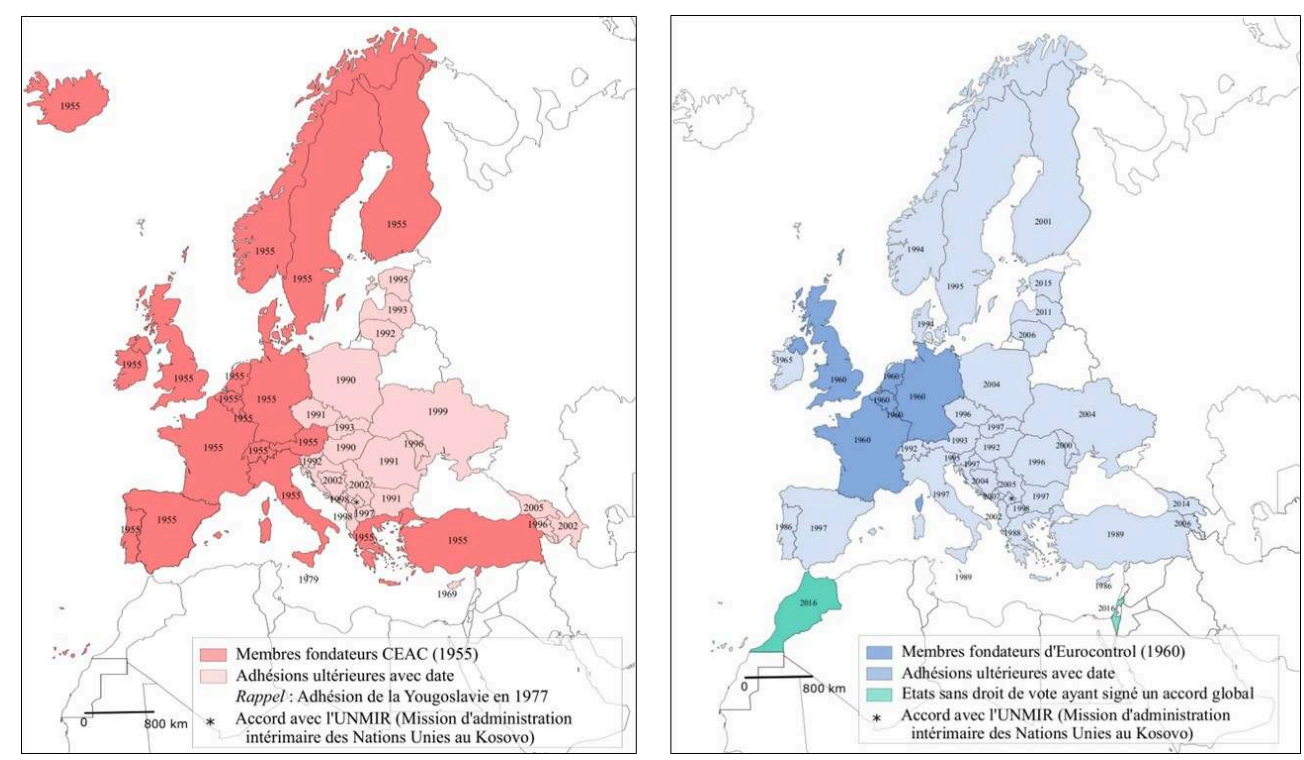

Source : CEAC et Eurocontrol

\section{Eurocontrol (1960), interprétations divergentes du contrôle aérien}

Eurocontrol peut être défini comme l'organisation européenne pour la sécurité de la navigation aérienne. A la fin des années 1950, l'adoption des avions à réaction par les compagnies aériennes civiles imposait un contrôle permanent et une intégration plus poussée des procédures dans la gestion des circulations aériennes. Elle conduisait à une réorganisation, tant pour l'accès à l'espace aérien supérieur, jusque-là réservé aux militaires que pour la définition de nouvelles routes et l'élargissement des aires de contrôle (McInally, 2010). L'urgence et l'ampleur des solutions à apporter militait pour un accord étroit et rapide entre un nombre restreint de pays, au premier plan desquels, les membres de l'OTAN directement concernés par l'intensification des trafics et une étroite coopération civile/militaire, dans le cadre du CIMIC (Coopération civile/ militaire) : l'Allemagne, la France (qui sortira du commandement intégré de l'OTAN en 1966), les trois pays du Benelux et le Royaume-Uni. Les six pays signeront la convention de 1960 (ratifiée en 1963) pour donner naissance à un organisme aux compétences techniques très affirmées: contrôle des flux aériens (définition des couloirs et allocation des trafics), collecte des redevances de navigation aérienne (en vigueur début 1971), établissement des prévisions de croissance du transport aérien en Europe. Un centre technique est installé en France, à Brétigny-sur-Orge, pour assurer la formation commune des contrôleurs aériens.

$\mathrm{Au}$ sein de cette structure, chaque pays-membre dispose d'une voix. Les recommandations sont prises à la majorité mais, pour être obligatoires, elles doivent être prises à l'unanimité. A sa création, deux lectures divergentes se sont opposées quant à l'ambition d'Eurocontrol. Certains pays assignaient à cet organisme la responsabilité d'une gestion directe et fédérale des mouvements aériens avec des moyens techniques dédiés et dirigés depuis le centre de Maastricht. Se rangeaient dans cette catégorie, la Belgique, les Pays-Bas, le Luxembourg et l'Allemagne. La France et le Royaume-Uni, dans une interprétation plus restrictive et soucieuse de la souveraineté nationale, attribuaient à Eurocontrol une fonction avant tout normative. 
L'amendement de 1981 leur donne finalement raison (McInally, 2010), conduisant Eurocontrol à recentrer son rôle sur la coordination, la planification des trafics, au détriment du contrôle direct, mais sans pour autant interdire cette option. Du projet fédéral demeure la zone MUAC (Maastricht Upper Air Control) qui met en œuvre un découpage transnational des espaces de contrôle et lui délèguent le recrutement et la gestion des contrôleurs aériens mutualisés (fig. 3). Si l'espace de contrôle de Maastricht reste géré selon de telles règles, les centres de Shannon et de Karlsruhe ont été renationalisés.

Figure 3. MUAC (Maastricht Upper Air Control).

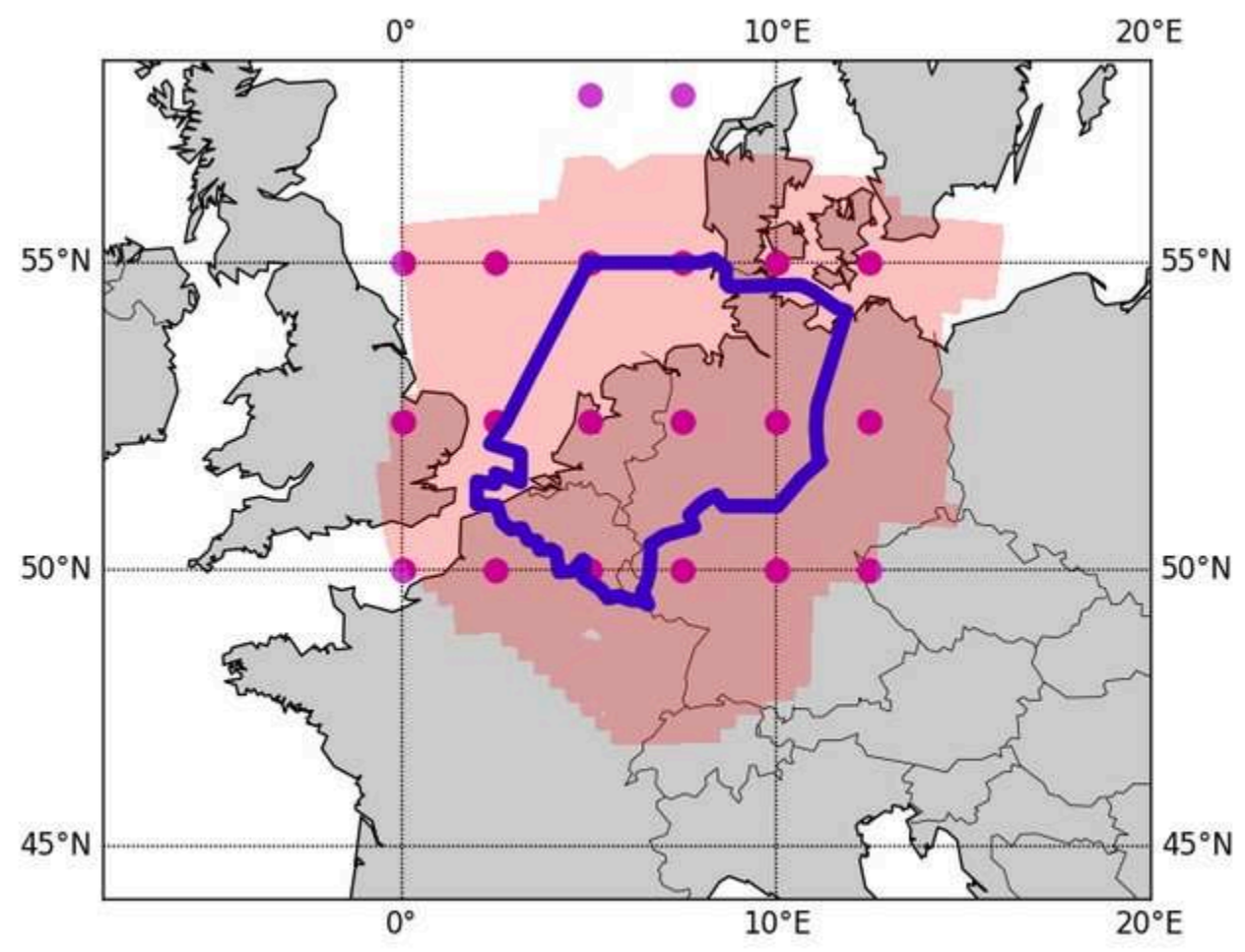

En bleu les limites de l'espace aérien MUAC ; en rouge la couverture radar et en violet les points de prévision météorologiques.

Source : Hoekstra, 2018

Les compétences techniques d'Eurocontrol et la position centrale des Etats membres de 1960 ont conduit d'autres pays européens à entretenir des liens étroits avec cette institution, sans pousser toutefois dans un premier temps jusqu'à l'adhésion. L'Irlande qui l'intègre en 1965, du fait de sa fonction de porte d'entrée européenne des vols transatlantiques reste une exception. La convention qui régit Eurocontrol est revue en 1997 dans un contexte de pression croissante des trafics aériens et dans la perspective d'élargissement politique de l'Europe. Elle permettait aussi de reconsidérer l'objectif de la structure qui se voit confier l'établissement d'un système de gestion unifiée des trafics aériens en Europe $\left(\mathrm{ATM}+{ }^{7}\right)$, dont la Commission Européenne s'est inspirée pour lancer son programme de Ciel Unique Européen. 
Les JAA (1970), l'aéronautique européenne à la recherche d'une taille critique

17 Les JAA ou Autorités conjointes de l'aviation ont été créées en 1970 pour constituer un organisme technique associé à la CEAC en 1978, sans être juridiquement parlant des organisations internationales. Regroupant jusqu'à 37 autorités réglementaires de l'aviation civile (soit à l'origine un rayonnement plus large que l'UE), les JAA permettaient à ces dernières de participer à l'élaboration des normes et de procédures communes (Joint Aviation Requirements ou JAR) dans la reconnaissance réciproque des matériels aéronautiques (airworthiness). L'objectif était de développer une certification standard commune avec en arrière-plan l'intérêt de l'industrie aéronautique européenne émergente (Airbus). Progressivement, la fonction a été élargie à la certification des aéronefs, à leur maintenance et l'homologation pour tous types d'avions. L'établissement de l'AESA (Agence européenne de la sécurité aérienne) en 2002, dotée d'un pouvoir réglementaire ayant force de loi à l'échelle de l'UE, a rendu cette fonction obsolète et conduit à supprimer les JAA en 2010 au profit d'une attribution restreinte au rôle de conseil dans le groupe AGNA (Advisory Group of National Authorities) rattaché à l'AESA (Cormier, 2010).

\section{L'AESA (2002), un pas supplémentaire vers l'intégration de l'espace aérien européen}

L'Agence européenne de sécurité aérienne (AESA) a été établie par l'Union Européenne en 2002 avec pour siège Cologne. Ses missions portent sur l'élaboration des règles techniques communes dans le domaine de l'aviation. Elle délivre les certifications pour les aéronefs et leurs composants, certifie les entreprises qui conçoivent, fabriquent et assurent l'entretien de produits aéronautiques. L'AESA est donc garante des normes de sécurité et de leur application dans tous les domaines de l'aviation civile pour l'Union européenne. Son influence industrielle est donc centrale pour le secteur. Afin de remplir sa mission, elle édicte des textes réglementaires communs qui s'imposent via les États-membres à tous les acteurs du secteur en Europe. En 2008, l'AESA voit ses attributions s'élargir au détriment des instances nationales avec la prise en charge de la conformité de gestion et de sécurité des aéroports ; les petits aérodromes restent de la compétence des États. C'est aussi à l'AESA qu'incombe la délivrance des licences communautaires de pilotes et les autorisations de circulation pour les aéronefs des pays tiers selon les critères prescrits par l'OACI (établissement des listes noires des compagnies aériennes). De manière plus générale, l'AESA a la responsabilité d'apporter un éclairage technique au législateur européen ainsi qu'aux États-membres.

\section{Les repositionnements institutionnels au sein d'un système en voie d'intégration}

\section{La mise en perspective du télescopage institutionnel}

19 Le paysage des institutions en charge de la gestion de l'espace aérien européen est donc composite et peut se lire comme une sédimentation de projets géopolitiques successifs voire concurrents. En principe, les États en occupent la place centrale en nouant des relations à divers niveaux et selon des intensités variables. Si la souveraineté nationale exclusive sur l'espace aérien demeure un principe, dans les faits, il est fortement 
atténué par de nombreuses coopérations inter-étatiques. Dernière venue, l'Union Européenne vient bousculer les anciens schémas par l'établissement d'un marché unique et d'une politique intégrative des transports. L'évolution de l'environnement politique, technique et commercial pousse à un réagencement régulier de la géographie des structures et des relations institutionnelles qu'elles entretiennent. Dans le cadre de l'UE, l'unification fonctionnelle de l'espace européen se fait par étapes avec les jalons décisifs que sont l'instauration de la libre concurrence (Acte unique européen de 1986) et à la mise en œuvre d'une politique commune (Traité de Maastricht de 1992). Elle accompagne la montée en puissance des structures communautaires dans la prise de décision conjointe institutions européennes/ État: "Les transports constituent une compétence partagée entre l'UE et les États-membres » (Art. 412 du Traité).

L'intégration de l'espace aérien européen s'opère simultanément sous deux aspects: celui du contrôle et celui des opérations commerciales. Le premier correspond à l'encadrement et de l'amélioration de la navigation aérienne, à travers le projet de "Ciel unique » qui cherche à dépasser la fragmentation territoriale pour améliorer l'efficacité du contrôle aérien. Plusieurs composantes sont mises en avant en association avec les pays de l'AELE: composante technologique avec SESAR ${ }^{8}$, opérationnelle avec l'établissement des $\mathrm{FAB}^{9}$ transnationaux, institutionnelle enfin avec un centre de commandement qui reste à réaliser. Le second aspect concerne l'établissement d'un marché intérieur complètement ouvert pour les services commerciaux aériens. Il se dessine entre 1993 et 1997 sur la base d'un progressif décontingentement des trafics jusqu'à l'ouverture complet des vols domestiques en cabotage. Le point central est que l'évolution rend caduc le principe des accords commerciaux intergouvernementaux posé par l'OACI entre pays de l'UE.

21 Cette évolution accompagne le bouleversement géopolitique que le continent traverse alors avec le retrait puis l'implosion de l'URSS suivi d'un double mouvement d'extension géographique et d'intégration des institutions européennes. La CEAC et Eurocontrol précèdent les phases successives d'élargissement de l'UE, doublées de l'approfondissement de ses compétences comme l'illustre la création de l'AESA en 2002. Les logiques communautaires se répercutent alors à trois niveaux : l'affirmation d'un marché unique via l'unification complète des normes, l'ouverture des prestations de service, la réorganisation du contrôle aérien sous une tutelle commune afin de répondre à des flux aériens croissants, enfin la centralisation des décisions prises dans la procédure de trilogue (Commission, Conseil de l'Union et Parlement).

22 S'impose progressivement la prééminence institutionnelle de l'UE dans les divers mécanismes de régulation de l'espace aérien européen. Pour ce faire, l'UE va mobiliser et réinterpréter les divers héritages des acquis réglementaires et des modes de fonctionnement existants des institutions pré-communautaires. Elle introduit des éléments qui réinterprètent et redynamisent en les renforçant les liens préexistants. Une telle évolution constitue un facteur de déstabilisation potentiel pour le système bermudien hors des frontières de l'UE, alors même que l'OACI ne fait toujours pas de place à l'UE, structure non-étatique et donc non inéligible dans cette instance et ce, malgré l'accroissement de son poids et son pouvoir de décision.

En outre, l'espace aérien européen ne se limite pas aux seuls membres de l'UE. Son tropisme institutionnel rayonne sur les États voisins avec lesquels elle a passé des accords qui ont pour effet de diffuser les règles communautaires au-delà des frontières des Etats-membres e bousculant les principes de Chicago (universalisme dans les 
normes et bilatéralisme souverain dans les relations économiques) au profit d'un multilatéralisme régional qui peut aussi s'interpréter par un bilatéralisme entre l'UE et ses partenaires.

\section{Les stratégies de régionalisation}

24 L'espace aérien européen tend à se réorganiser autour de sa composante communautaire qui en devient le noyau. Dans cette perspective, trois juristes (Loïc Grard, 2003 ; Mathieu Vaugeois, 2014 ; Vincent Correia, 2014) ont particulièrement travaillé sur la question aérienne pour comprendre comment les logiques communautaires viennent perturber les règles internationales. En d'autres termes, ils analysent comment se construit progressivement une régionalisation du droit, entre l'échelle internationale (universalité des règles techniques) et les accords bi-étatiques (souveraineté des droits aériens commerciaux). Il nous faut comprendre les mécanismes par lesquels cette logique peut s'affirmer.

Le Traité de Rome de 1957 qui posait une politique commune des transports en excluait l'aérien et le maritime. En effet, l'étroitesse de la CEE à 6 et le fonctionnement satisfaisant $\mathrm{du}$ cadre international existant expliquait sans doute cette retenue (Stevens, 2004). D'ailleurs, même pour le transport terrestre, les dispositifs visés vont rester lettre morte jusqu'en 1986 (Versini, Decoster, 2009). L'Acte unique de 1986 et la perspective de l'achèvement du marché intérieur au 1/1/1993 vont en revanche profondément changer la donne avec l'adoption du principe de libre prestation de service commercial aérien, en d'autres termes avec l'instauration de la libre concurrence dans l'UE. Le célèbre arrêté dit « Nouvelles Frontières " (CJCE 30/4/1986) rendu le 30 avril 1986 condamnait en effet, comme contraire au droit communautaire, la pratique établie d'encadrement tarifaire de l'IATA (association des compagnies aériennes) avec l'accord des gouvernements. L'ouverture par étape à la concurrence via divers paquets aériens aboutit à l'effacement des restrictions et de fait à l'obsolescence des traités bilatéraux dans un cadre intra-communautaires. En 1997, les neuf libertés aériennes étaient acquises au sein de l'UE pour les compagnies aériennes immatriculées dans un État-membre, y compris donc les vols domestiques dans chacun des pays.

Cette évolution questionnait par contrecoup les règles classiques du droit international aérien fondées sur la souveraineté complète des État, menant l'UE à mobiliser deux principes relevés par Loïc Grard (2003) :

- La théorie des compétences implicites qui pose que laisser aux États-membres la compétence de négocier des accords aériens internationaux hors UE «peut affecter substantiellement les règles communes déjà adoptées. Ainsi, les États-membres doivent s'effacer au profit de l'UE ».

- La théorie des compétences ascendantes qui infère qu'au-delà du marché unique de l'aviation commerciale, la concurrence non faussée ne peut devenir une réalité pérenne sans une unification des règles de protection de la sécurité, de l'environnement ou du travail ; vouloir le marché unique c'est donc aussi étendre l'homogénéité des règles en vigueur dans d'autres champs, selon le principe de la concurrence non faussée.

En effet, le principe de la libre négociation bilatérale entre États-membres et État-tiers de l'OACI permettait à certaines compagnies aériennes d'obtenir des avantages concurrentiels en contradiction avec l'égalité de traitement que posait le marché unique. Concrètement, les accords nationaux d'open skies entre les États-Unis et les 
Pays-Bas ou le Royaume-Uni favorisaient les échanges bilatéraux au détriment de leurs concurrents européens. De leur côté, les compagnies américaines, grâce à la $5^{\text {ème }}$ liberté ${ }^{10}$ étaient en mesure d'assurer un service intra-UE sans réciproque sur leur marché domestique, puisque, seules dans le droit aérien les premières sont considérées comme internationales. Il existait donc un "hiatus entre le caractère intégré du marché intérieur dans sa dimension interne et sa fragmentation au plan externe" (Correia, 2014). La préservation de l'unicité du marché amène la Cour de Justice des Communautés européenne à trancher. Les arrêts dits "Open Skies" de novembre 2002 viennent consacrer la compétence de l'UE comme unique mandataire des négociations avec les pays tiers (Grard, 2004). Il faut toutefois ménager une subtilité juridique : l'UE n'étant pas reconnue dans le droit aérien international et le traité de Rome n'engageant que États signataires et non les tiers, les accords aériens reposent formellement sur des accords conjoints qui retiennent une participation superposée et non substituée de l'UE et des États-membres (Correia, 2014), et cela même si in fine les négociations sont menées par la Commission. Juridiquement, l'Union coexiste avec les États-membres, seuls sujets originaires dans l'ordre international. On a ainsi pu parler « d'union of sovereign airspaces " ou "d'espace normatif européen" (Correia, 2014) et non de territoire, terme réservé aux États. En tout cas, la relation instaurée glisse d'un bilatéralisme inter-étatique à un multilatéralisme régional, dénationalisant de fait le droit aérien au sein de l'UE.

Par ailleurs, le souci de coordination ne s'arrête pas aux limites de l'UE. Outre les pays de l'Espace économique européen, des négociations ont été engagées avec les pays proches dans le cadre de la politique de voisinage (Géorgie, Moldavie, Ukraine) au sein de EACE (Espace aérien commun européen), mais aussi avec Israël, le Maroc ${ }^{11}$ ou la Jordanie dans le cadre d'association avec les États de l'Union pour la Méditerranée. Les deux approches conduisent à déployer chez les signataires les règles et les standards intra-communautaires conduisant de fait à l'exportation du droit et des normes de l'UE en contrepartie de l'accès à son marché. Cette recherche d'alignement du cadre réglementaire est aussi un argument introduit par l'UE dans les négociations de libéralisation des espaces aériens avec le Canada ou le Brésil, ou la négociation en cours d'un accord d'open skies avec l'ASEAN, ce qui serait alors le premier accord interrégional. Ces négociations se traduisent par des comités techniques mixtes, distinguant l'open aviation area, méthode européenne qui repose sur un approfondissement des cadres du marché à travers la coopération technique et normative avec la prise en compte des dimensions sociale ou environnementale, de l' open skies américaine, plus compatible avec les règles bermudiennes car limité aux seules questions d'accès au marché, de capacité et de tarifs (Correia, 2014). 
Figure 4. Le schéma interprétatif du réagencement des acteurs institutionnels de l'espace aérien européen.

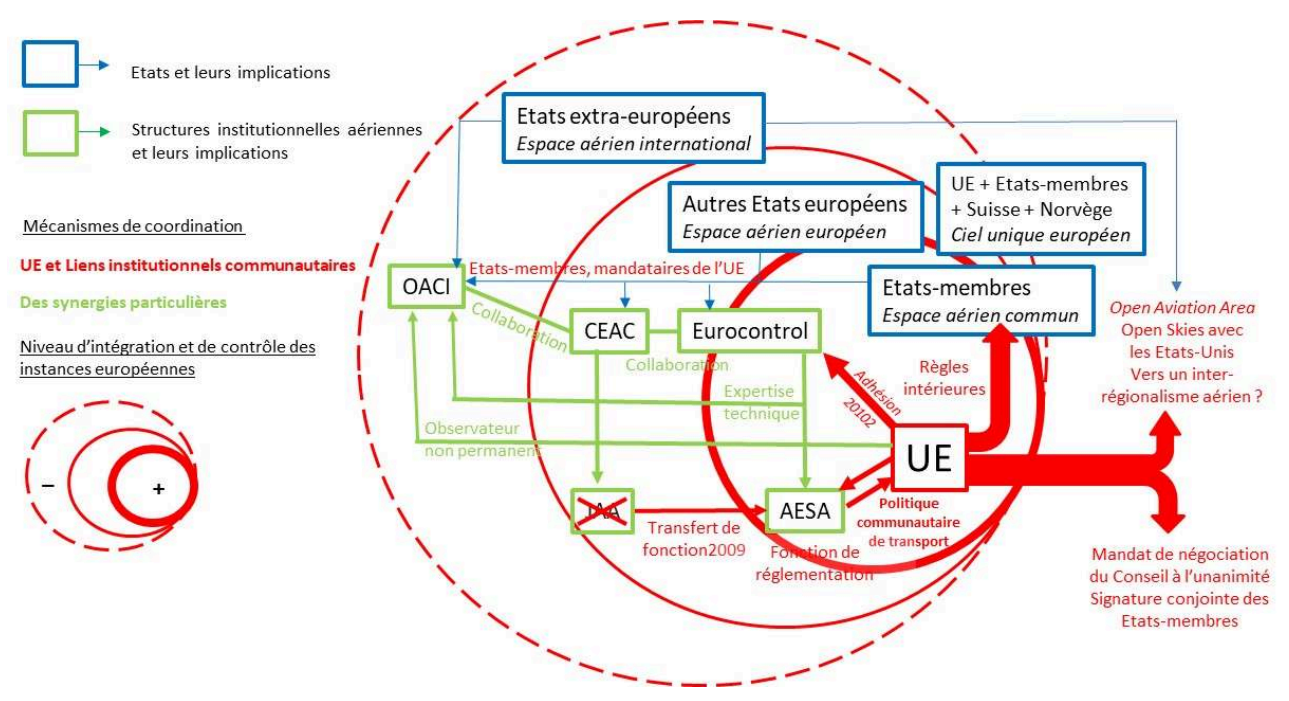

Conception : A. Beyer

\section{La réévaluation des rapports entre institutions aériennes}

Comment l'UE est-elle alors prise en compte par les institutions aériennes internationales? Comment sa montée en puissance singulièrement renforcée par son mandat de négociation au nom des Etats-membres et l'affirmation de ses compétences réglementaires vient-elle bousculer l'ordre institutionnel existant ?Par ses statuts, on l'a vu, l'OACI n'est susceptible d'accueillir comme membres que des États souverains. A ce titre, l'UE dispose d'un statut d'observateur invité à l'OACI, mais sans droit de vote, ni de la possibilité de présenter ou d'appuyer des motions. En revanche, son représentant peut intervenir en séance plénière et participer aux groupes de travail. Ainsi, bien qu'acteur de poids à l'échelle mondiale, l'UE ne peut prétendre peser directement dans la définition des normes aériennes internationales ou défendre des positions politiques et techniques, alors même qu'elle dispose d'une expertise reconnue (Vaugeois, 2014). Les membres non-européens ne sont guère favorables à lui accorder un siège. Ce serait une voix supplémentaire pour l'Europe, une région du monde déjà bien représentée à l'OACI. A l'inverse, substituer les représentants des Etats-membres par un délégué unique pour l'UE, ferait perdre l'avantage numérique dans les instances et les diverses commissions. Aussi, le statut quo est aujourd'hui conservé. Outre la concertation interne, un des États-membres est en charge de porter plus spécifiquement le point de vue de l'UE au sein de l'OACI, d'autant que cette dernière est loin d'être défavorable au régionalisme aérien. Aujourd'hui, lors des votes à l'OACI, les intérêts communautaires sont donc défendus par les États-membres, ceux qui disposent d'un siège permanent ou ceux qui y sont représentés au sein de pools (Scandinavie ou Europe Centrale). Son poids est donc déjà plus important qu'Eurocontrol, membre invité comme observateur depuis 1964. La menace d'un unilatéralisme européen au sein de l'OACI est-il alors fondé ? Il semblerait que ce ne soit pas le cas. Ainsi, dans les domaines qui la concerne, l'UE se soumet en effet aux règles internationales de l'OACI et les impose à ses États-membres et à ses partenaires, en assurant ainsi une plus rapide et une plus homogène diffusion (Correia, 2014). 
Toutefois, la directive de 2008 (2003/87/CE) avait donné un signal inverse. Elle soumettait de manière unilatérale à une taxe les émissions de gaz à effet de serre pour les compagnies, européennes ou non-européennes, pour tous les vols touchant le territoire européen. Cette mesure a été fermement combattue par les autres pays comme abusive et finalement condamnée par l'OACI en 2013 et retirée par la Commission. Ce recul technique et diplomatique rappelle la faiblesse de l'influence communautaire dans une instance dont nombreux sont les membres qui la considèrent comme une simple instance régionale, privée de vocation internationale (Compagnon, 2016).

30 Le développement normatif et opérationnel de l'UE dévolu à l'AESA accroît par ailleurs les risques d'interférences avec les institutions régionales déjà en place. Plusieurs arrangements sont alors possibles pour éviter des redondances, sources potentielles de conflits. En 1997, la redéfinition de la convention d'Eurocontrol a permis à l'UE une adhésion de plein droit, alors qu'elle n'est qu'observatrice à la CEAC depuis 1980, dupliquant en cela sa position à l'OACI.

Un partage des rôles s'est progressivement établi où l'AESA à vocation réglementaire s'appuie sur Eurocontrol pour ses évaluations techniques (contrôle aérien et planification du trafic). L'expertise d'Eurocontrol est en fait sollicitée depuis longtemps par la Commission européenne : dès 1977 pour les études, puis régulièrement à partir des années 1980 dans l'élaboration de stratégies. Sa position comme ressource technique s'affirme encore en 2008, avec l'élargissement des prérogatives de l'AESA dans la gestion du trafic aérien. La spécificité des compétences techniques développées par Eurocontrol et qui aurait pu lui être retirée, conduit au contraire l'Agence européenne à développer avec elle un partenariat étroit. Non seulement Eurocontrol est maintenu, mais il se retrouve encore renforcé dans certaines de ses activités (établissement des tarifs et collecte des redevances en route). Eurocontrol est devenu un maillon essentiel dans la définition de certaines directives qui exigent la connaissance fine des données de trafic. Regroupant 38 pays, cette institution déborde néanmoins le périmètre strict de l'UE et trouve ainsi naturellement une fonction d'harmonisation entre les règlements communautaires de AESA et les 11 pays tiers mais indirectement influencés par l'AESA. 
Figure 5. De l'architecture des institutions à l'emboîtement régional des espaces aériens. Schéma cartographique de synthèse.

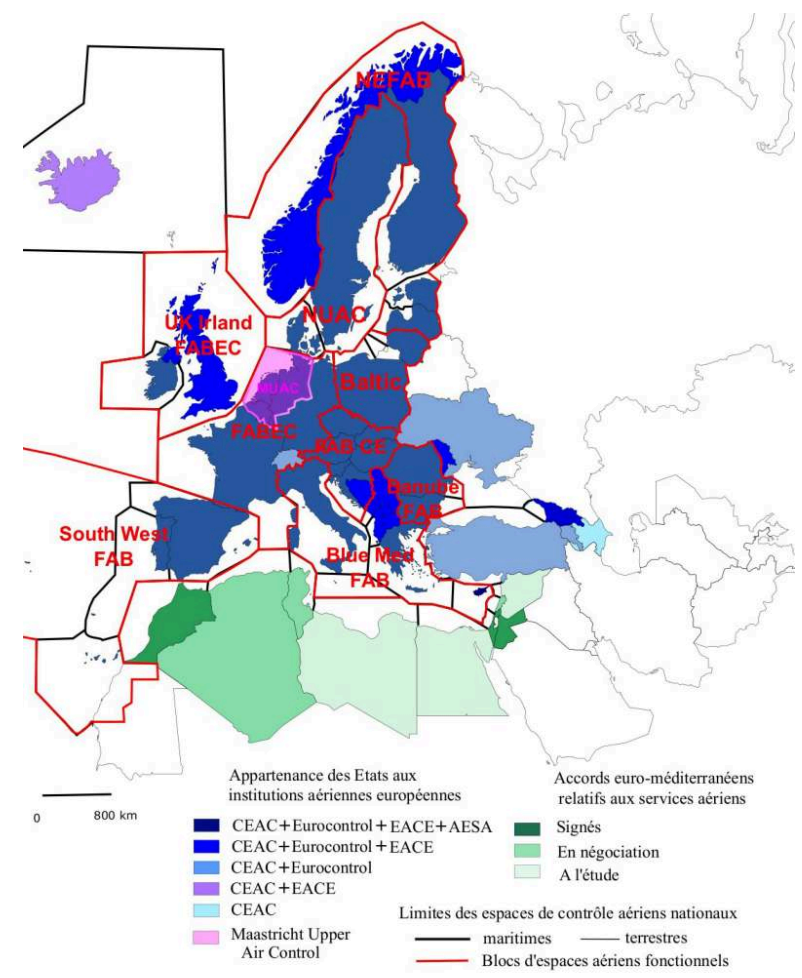

Réalisation : A. Beyer - sources diverses

\section{Conclusion : La contribution du droit international à la géographie des gouvernances régionales des espaces aériens}

La suppression du cadre bilatéral des accords de desserte aérienne, via le principe du marché unique et la totale liberté de desserte s'est imposée aux États-membres assurant l'ouverture au multilatéralisme à la composante supranationale dans les relations commerciales et réglementaire. Cette logique se diffuse désormais dans les relations extra-européennes, où l'UE est appelée à négocier des traités bilatéraux en substitution des États-membres et sous leur contrôle. Elle vient par-là bousculer l'ordre international de l'aviation civile, qui de fait n'est plus strictement interétatique. D'un ordre bilatéral, l'évolution conduit à la reconnaissance encore partielle et contestée d'un régionalisme d'intégration dans l'espace aérien international. Celui-ci s'organise selon un emboîtement qui mettent en lumière la construction juridique des espaces et leur portée territoriale, au sens plein du terme d'exercice de la souveraineté étatique. Dans cette perspective, l'analyse suivie montre tout l'intérêt euristique de la compréhension approfondie des relations entre les institutions internationales pour éclairer les dynamiques de territorialisation régionale.

La politique aérienne libérale de l'UE repose sur une conditionnalité réglementaire stricte exigeant un alignement normatif de la part des Etats-membres et de ses partenaires. Par le biais à la fois des institutions auxquelles adhèrent les pays-membres 
et qui débordent de l'espace de l'UE ou via les négociations, elle peut peser de manière décisive dans la définition des normes compatibles et souvent dérivées du contexte communautaire. Ainsi, les effets de la création d'un marché intérieur ont aussi une portée directe sur l'organisation des relations avec les pays tiers, élargissant la portée de la régionalisation de l'UE en dehors de son périmètre immédiat, ce que l'on pourrait qualifier de «halo territorial ». L'espace aérien européen se conçoit comme autant de cercles concentriques qui reprennent l'idée d'une géométrie variable. L'apport de la lecture juridique permet de bien comprendre la nature des relations qui lient ces espaces et précisent la nature des relations qu'entretiennent les institutions, véritable armature du système. Ces différents accords articulent des échelles qui dépassent le cadre de l'UE et de ses États-membres tout en redéfinissant en interne les cadre du contrôle aérien avec les FAB. Pour mettre en perspective l'analyse de l'UE, il faut bien sûr engager l'analyse d'autres ensembles régionaux sous l'angle de la constitution de leurs espaces aériens pour comprendre la nature des relations entre les États et leur degré d'intégration. Enfin, si les accords aériens entre ensemble régionaux restent encore rares, celui initié entre l'UE, l'ASEAN ou l'UEMOA en 2009 pourrait assurément inaugurer un nouveau type de régulation internationale dans le domaine. Abordée ici au travers du secteur du contrôle aérien, l'article montre tout l'intérêt qu'il peut y avoir d'aborder les espaces régionaux sous l'angle des relations entre acteurs institutionnels. L'approche croisant la géographie, le droit et l'histoire permet de préciser les articulations entre les espaces de gestion emboîtés et partant offre une méthode pour analyser les processus de territorialisation à l'œuvre et d'en comparer l'intensité.

\section{BIBLIOGRAPHIE}

AGNEW J. (2005), « Sovereignty Regimes: Territoriality and State Authority in Contemporary World Politics ", Annals of the Association of American Geographers, 95, 2, pp. 437-461.

AGNEW J. (2009), Globalization and Sovereignty, beyond the territorial trapp, Lanham, Rowman \& Littlefield, $231 \mathrm{p}$.

BEYER A. (2008), « Anciennes et nouvelles frontières du Ciel Unique Européen. Enjeux techniques et territoriaux de la navigation aérienne en Europe », Flux, 71, pp. 8-23.

BEYER A. (2014), La construction d'un espace européen des transports. Du principe de souveraineté nationale aux modalités de son dépassement, HDR Volume 3, texte original, 319 p., en ligne.

BEYER A. (2019), « La gestion du corridor ferroviaire Rhin-Alpes, une mise en pratique de la construction européenne par les transports ", Géoconfluences, en ligne.

BOWEN J. (2010), The Economic geography of Air Transportation. Space, time and the freedom of the Sky, New York, Routledge, 333 p.

BLAVOUKOS S., BOURANTONI D. (dir.) (2010), The EU Presence in International Organizations, Routledge advances in European Politics, London \& New York, Routledge, 186 p. 
BLOMLEY N.K. (1994), Law, Space, and the Geographies of Power, New York \& London, The Guilford Press, $259 \mathrm{p}$.

COMPAGNON D. (2016), « Expliquer la “crise” entre l'UE et l'OACI sur la réduction des émissions de gaz à effet de serre dans l'aviation internationale », Études internationales, 47, 2-3, pp. 263-283.

CORMIER H. (2010), « Sécurité aérienne. L'Europe se prépare à 2013 », Aviation Civile Magazine, 354, octobre, pp. 11-15.

CORREIA V. (2014), L'Union Européenne et le droit international de l'aviation civile, Bruxelles, Bruylant, $970 \mathrm{p}$.

DEBBAGE K. (2014), “The Geopolitics of Air Transport”, in GOETZ A, BUDD L., The Geographies of Air Transport, Farnham, Ashgate, coll. Transport and Mobility Series, pp. 25-39.

DIEZ DE VELASCO VALLEJO M. (2002), Les organisations internationales, Paris, Economica, 919 p.

DOBRUSZKES F. (2014), “Geographies of European Air Transport”, in GOETZ A., BUDD L. (2014), The Geographies of Air Transport, Farnham, Ashgate, coll. Transport and Mobility Series, pp. 167 186.

FOREST P. (ed.) (2009), Géographie du droit. Épistémologie, développements et perspectives, Québec, Presses de l'Université Laval, 296 p.

FOREST P. (2015), « Vers une géographie du droit ? Pistes de réflexion », Développement durable et territoires, 6, 1 .

FRÉTIGNY J.-B. (2013a), « La frontière à l'épreuve des mobilités aériennes : étude de l'aéroport de Paris Charles-de-Gaulle », Annales de géographie, 2, 690, pp. 151-174.

FRÉTIGNY J.-B. (2013b), Les mobilités à l'épreuve des aéroports : des espaces publics aux territorialités en réseau. Les cas de Paris Roissy-Charles-De-Gaulle, Amsterdam Schiphol, Francfort-sur-le-Main et Dubai International, Thèse de géographie, Dir. N. Cattan, Université Paris 1, 659 p.

GRARD L. (2003), «L'UE et le droit international de l'aviation civile », Annuaire français de droit international, 49 , pp. 492-515.

GRARD L. (2004), L'Europe des transports, Actes du colloque d'Agen, 7 et 8 octobre 2004, Paris, La Documentation française, $857 \mathrm{p}$.

GUINCHARD M. (1961), « La coopération entre les États pour le contrôle de la circulation aérienne », Annuaire français de droit international, 7, pp. 450-463.

HENRICH-FRANKE C. (2007a), « Europäische Verkehrsintegration im 19. und der zweiten Hälfte des 20. Jahrhunderts », in HENRICH-FRANKE C., NEUTSCH C., THIEMEYER G., Internationalismus und Europäische Integration im Vergleich. Fallstudien zu Währung, Landwirtschaft, Verkehrs- und Nachrichtenwesen, Nomos, Baden-Baden, pp. 133-175.

HENRICH-FRANKE C. (2007b), "From a Supranational Air Authority to the Founding of the European Civil Aviation Conference (ECAC)”, Journal of European Integration History, 1.2007, pp. 69-90.

HENRICH-FRANKE C. (2008), "Mobility and European integration. Politicians, professionals and the foundation of the ECMT", The Journal of Transport History, 1.2008, pp. 64-82.

McINALLY J. (2010), Eurocontrol History Book, Cologne, Eurocontrol, 304 p.

MARC N. (2014), Enjeux d'appropriation de l'espace aérien en France et en Europe : vers une territorialisation spécifique, Thèse de géographie, Dir. Vincent Veschambre, Université d'Angers, $315 \mathrm{p}$. 
PELSSER A. (2019), The Postal History of ICAO, https://applications.icao.int/postalhistory/ index.html.

RICHARD Y., MAREÏ N. (coord.) (2018), Dictionnaire de la régionalisation du monde, Atlande.

SANGUIN L. (1977), « Géographie politique, espace aérien et cosmos », Annales de géographie, 86, 475, pp. $257-278$.

SCHOT J. (2010), “Transnational infrastructures and the Origin of European Integration”, in BADENOCH A., FICKERS A. (eds.), Materializing Europe. Transnational Infrastructures and the Project of Europe, Palgrave Macmillan, pp. 82-109.

SCHOT J., SCHIPPER F. (2011), “Experts and European transport integration, 1945-1958”, Journal of European Policy, 2, pp. 274-293.

STEVENS H. (2004), Transport Policy in the European Union, Houndmills, Palgrave, The European Union Series, $276 \mathrm{p}$.

STRALE M. (2006), « Géographie mondiale des alliances de compagnies aériennes », Belgeo, 4, https://journals.openedition.org/belgeo/11761.

THÉRY H. (2017), « Les ailes de la centralité, réseaux aériens planétaires et mondialisation », Mappemonde, 119, pp. 1-19.

MISA T.J., SCHOT J. (2005), “Technology and the Hidden Integration of Europe. Introduction”, History and Technology, 21, 1, pp. 1-19.

VAN DER FLEUTEN E., KAIJSER A. (2005), “Networking Europe”, History and Technology, 21, 1, pp. 21-48.

VAUGEOIS M. (2014), Vers une adhésion de l'EU à l'OACI ?, Mémoire de master 2, Institut de droit aérien et spatial, Faculté de droit, Université McGill, Montréal.

VERSINI F., DECOSTER F. (2009), UE : la politique des transports. Vers une mobilité durable, Paris, La Documentation française, $154 \mathrm{p}$.

VOWLES M.T. (2006), “Geographic Perspectives of Air Transportation”, The Professional Geographer, 1, pp. 12-19.

\section{$\underline{\text { Liste des acronymes }}$}

AESA : Agence européenne de sécurité aérienne

AGNA : Advisory Group of National Authorities

ATM : Air Traffic management

CEAC : Conférence Européenne de l'Aviation Civile

CIMIC : Coopération civile/militaire

CINA : Commission internationale de Navigation Aérienne

CJCE : Cour de Justice de l'Union européenne des Communautés européenne, ancienne appellation de l'actuelle Cour de justice de l'Union européenne.

EANP : European Navigation Planning Group

IATA : Association internationale du transport aérien

JAA : Joint Aviation Authorities ou Autorités conjointes de l'aviation

JAR : Joint Aviation Requirements

EACE : Espace aérien commun européen

EANP : European Navigation Planning Group

FAB : Functional Airspace Block

IATA : International Air Transport Association

MUAC : Maastricht Upper Air Control 
OACI : Organisation de l'aviation civile internationale

PIRGs : Planning and Implementation Regional Groups

UEMOA : Union Economique et Monétaire Ouest Africaine

UE : Union européenne

\section{NOTES}

1. Les acronymes sont précisés lors de leur première mention et recensés à la fin de l'article.

2. On peut mentionner ici la Conférence Aéronautique Internationale (Europe de l'ouest et Europe Centrale) (1920), à la suite des Conférences anglo-franco-belges, la Conférence aéronautique méditerranéenne (1930) ou la Conférence aéronautique des États baltiques et des Balkans (1934). Des États non-membres de la SDN ont mis sur pied leurs propres accords aériens internationaux dans l'hémisphère occidentale, autour de l'Espagne (Convention ibéro-américaine de 1926) et autour des États-Unis (Convention de La Havane de 1928). La Commission Aéronautique Permanente pour l'Amérique, initiée dès 1916 par la Conférence panaméricaine est pour sa part restée à l'état de projet (Pelsser, 2019).

3. À noter cependant l'existence de l'Accord relatif au Transit des Services Aériens Internationaux (ou accord sur le transit aérien) qui stipule que les droits de 1ère liberté de l'air (survol) et de 2e liberté de l'air (escale technique) sont automatiquement échangés entre les pays signataires de cet accord (actuellement 135 signataires).

4. SARP : Standards And Recommended Practices.

5. "The Council may, where appropriate and as experience may show to be desirable, create subordinate air transport commissions on a regional or other basis and define groups of states or airlines with or through which it may deal to facilitate the carrying out of the aims of this Convention ".

6. Le Conseil de l'Europe est une organisation régionale intergouvernementale créée en 1949 à Strasbourg. Elle ne doit pas être confondue avec les structures communautaires que sont le Conseil de l'Union européenne regroupant les ministres des Etats-membres de l'UE ou le Conseil européen qui en regroupe les chefs d'Etat.

7. ATM : Air Traffic Management.

8. Single European Sky Air traffic management Research

9. FAB Functional Airspace Block ou Bloc d'espace aérien fonctionnel, délimités en fonction des besoins du trafic aérien et non des frontières d'États.

10. Cinquième liberté de l'air : droit ou privilège accordé par un État à un autre État, dans le contexte de services aériens internationaux réguliers, de débarquer et d'embarquer, dans le territoire du premier État, du trafic en provenance ou à destination d'un État tiers. Cette liberté permet de rentabiliser certaines routes, en permettant aux avions de ne pas repartir à vide.

11. A noter que le contrôle aérien du Sahara Occidental est assuré par l'Espagne mandaté par les Nations Unies depuis les îles des Canaries et non par le Maroc. La Cour de justice de l'Union européenne exclut le Sahara Occidental de l'accord sur l'aviation civile, conclu entre l'UE et le Maroc. La position espagnole est par ailleurs contestée par le Front Polisario qui revendique la pleine indépendance du territoire au profit des population sarahouies. La CJUE a indiqué que dans le cadre où les transactions commerciales concernent le Sahara occidental, les pays membres de l'UE doivent traiter avec le représentant le Front Polisario. 


\section{RÉSUMÉS}

La lecture de l'espace aérien européen souligne tout le parti qui peut être tiré d'une approche juridique et historique de la construction des espaces régionaux et de leurs géographies. Le propos de l'article est ainsi de voir comment l'intégration européenne tend à redistribuer de manière dynamique le système institutionnel en charge de la gestion de l'espace aérien européen et de comprendre comment « l'ascendance croissante de l'EU » (Blavoukos, 2011) vient bousculer et réinterpréter divers héritages. Les principales organisations internationales du secteur (OACI, CEAC, Eurocontrol, AESA) opèrent à des échelles et selon des cadres différenciés et évolutifs. Elles s'inscrivent dans les séquences d'une histoire longue dont elles portent les marques. Mais par ailleurs, elles font preuve d'une forte capacité d'adaptation systémique dont la seule cartographie aurait bien du mal à rendre compte. Mais l'enjeu demeure bien géographique avec pour but l'organisation de l'espace aérien commun et du partage de sa souveraineté.

A legal and historical approach of European airspace highlights the advantage taken by observing the construction of regional entities from a geographer's perspective. The European integration sheds a new light on the various historical and institutional legacies as they are involved in a dynamic system marked by "growing ascendancy of the EU" (Blavoukos, 2011). The main international organizations in charge of the European air space management (ICAO, ECAC, Eurocontrol, EASA) are operating at several geographical scales in an evolving geopolitical context that cartography on its own would have a hard time to render properly. However, such a challenge has an obvious geographical dimension in organizing a common airspace and in sharing the national sovereignties over it.

\section{INDEX}

Keywords : air space, regional integration, European Union, ICAO, ECAC, Eurocontrol, EASA Mots-clés : espace aérien, intégration régionale, Union Européenne, OACI, CEAC, Eurocontrol, AESA

\section{AUTEUR}

\section{ANTOINE BEYER}

Professeur des universités, Université de Cergy-Pontoise, antoine.beyer@u-cergy.fr 